\title{
The framework of P systems applied to solve optimal watermarking problem
}

\author{
Hong Peng ${ }^{a}$, Jun Wang ${ }^{b}$, Mario J. Pérez-Jiménez ${ }^{c}$, Agustín Riscos-Núñez $^{c}$ \\ ${ }^{a}$ Center for Radio Administration and Technology Development, Xihua University, Chengdu 610039, China \\ ${ }^{\mathrm{b}}$ School of Electrical and Information Engineering, Xihua University, Chengdu 610039, China \\ ${ }^{\mathrm{c}}$ Research Group of Natural Computing, Department of Computer Science and Artificial Intelligence, University of Seville, Sevilla 41012, Spain
}

Keywords:

Membrane computing

P systems

Image watermarking

Optimal watermarking problem
A B S T R A C T

\section{Introduction}

Digital watermarking has became one of the most effective tools for copyright protection of digital media (such as image, audio, video) [1-4]. A large number of watermarking methods or algorithms have been addressed in recent years, such as adaptive watermarking methods [5,6], quantization-based watermarking methods [7-9], machine learning-based watermarking methods [10-15]. It is well-known that imperceptibility and robustness are widely used to measure the performance of a watermarking system. Imperceptibility indicates perceptual quality of the media data being protected. Usually, the embedded watermark should be invisible and over all media types. Meanwhile, the watermark should be also robust to signal processing or attacks. Ideally, the amount of signal distortion necessary to remove the watermark should degrade the desired media quality to a point of becoming commercially valueless. However, imperceptibility and robustness are conflicting with each other. Therefore, we have to balance the pair of conflicting performance measures efficiently. From the viewpoint of watermark embedding, watermarking parameters (such as embedding strength and threshold) and/or embedding position are crucial factors of effecting both imperceptibility and robustness. This rises an interesting and open problem, known as optimal watermarking problem. Optimal watermarking problem means how to find the optimal watermarking parameters and/or most suitable embedding positions for a watermarking algorithm such that its imperceptibility and robustness can be balanced optimally. In recent years, genetic algorithm (GA) has been introduced to 
investigate this problem and a number of optimal watermarking schemes have been addressed [16-19]. In summary, the role of GA in solving the optimal watermarking problem is mainly reflected in the following two aspects: (i) it searches most suitable frequency bands for watermark embedding [16]; (ii) it searches the optimal watermarking parameters for a watermarking algorithm such that both imperceptibility and robustness can be balanced optimally [17-19]. However, some deficiencies in GA performance can lead to the degradation in efficiency of these watermarking schemes, such as the premature convergence. This paper will focus on the optimal image watermarking problem and explore its solution under the framework of P systems.

Membrane computing initialed by Pǎun in 2000 [21], known as P systems, is a novel class of distributed parallel computing models, which was inspired by the structure and functioning of living cells as well as the interactions of living cells in tissues or higher order biological structures. A P system usually consists of three ingredients: membrane structure, multisets of objects and evolution rules [22]. The multisets of objects are placed in compartments surrounded by membranes, and evolved by some given rules that are applied in a non-deterministic maximally parallel manner (see [21] for details). The cell P systems were first investigated, where their membranes were arranged as a tree-like structure. Another kind was neural $\mathrm{P}$ systems in which spiking neural P systems were widely discussed in theory in recent years [23-26]. Tissue P systems focused on by this work were inspired by the intercellular communication and cooperation among neurons [27]. The communication among cells is based on symport/antiport rules. The tissue $\mathrm{P}$ systems can be viewed as a net of processors dealing with symbols and communicating them along channels specified in advance. Over recent years, a large number of $\mathrm{P}$ systems and variants have been proposed [22], which have addressed the synchronous and non-deterministic properties and parallel computing advantage of $\mathrm{P}$ systems.

This paper introduces tissue $\mathrm{P}$ systems to discuss the optimal watermarking problem. We first propose a ridgeletbased watermarking algorithm, which modulates ridgelet coefficients on strongest energy direction using an energy modulation technique, and then develop a novel watermarking framework based on tissue $\mathrm{P}$ systems with symport/ antiport rules. The reason that the framework can effectively exploit the optimal thresholds for the watermarking algorithm is due to three aspects: (i) co-evolution mechanism of objects between multiple membranes; (ii) the diversity of objects in the system; (iii) parallel computing ability.

This paper is organized as follows. Section 2 reviews basic definition of tissue $\mathrm{P}$ systems, and Section 3 gives a basic image watermarking algorithm used in this paper. Section 4 describes the proposed framework of solving the optimal watermarking problem. Experimental results are provided in Section 5, and conclusions are discussed in Section 6.

\section{Tissue P systems with symport/antiport rules}

Since the basis of solving optimal watermarking problem in this paper is using a tissue P system with symport/antiport rules, we briefly review its definition and mechanisms in this section. More detailed descriptions of tissue P systems can be found in [22,27-29].

Formally, a tissue $\mathrm{P}$ system (of degree $d>0$ ) with symport/antiport rules is a construct:

$\Pi=\left(O, w_{1}, \ldots, w_{d}, R_{1}, \ldots, R_{d}, R^{\prime}, i_{0}\right)$

where

(1) $O$ is a finite alphabet, whose symbols are called objects;

(2) $w_{i}(1 \leq i \leq d)$ is finite set of strings over 0 , which represents multiset of objects initially present in cell $i$;

(3) $R_{i}(1 \leq i \leq d)$ is finite set of evolution rules in cell $i$;

(4) $R^{\prime}$ is finite set of communication rules of the form $(i, u / v, j)$, which represents communication rule between cell $i$ and cell $j, i \neq j, i, j=1,2, \ldots, d, u, v \in O^{*}$;

(5) $i_{0}$ indicates the output region of the system.

From membrane structure, a tissue $\mathrm{P}$ system can be viewed as a net implicitly, which consists of the $d$ cells labeled by $1,2, \ldots, d$. Here, each cell is an elementary membrane. Usually, the environment is labeled by 0 . The communication rule of the form $(i, u / v, j)$ indirectly indicates synaptic connection between cell $i$ and cell $j$. The communication rules determine a virtual graph, where the nodes are the cells and the edges indicate if it is possible for pairs of cells to communicate directly. The net structure provides the flexibility of expressing the needed structures from simple to complex when we deal with real-world problems.

In tissue $\mathrm{P}$ systems, multisets of objects of the $d$ cells are described by $w_{1}, w_{1}, \ldots, w_{d}$. Suppose any multiset of objects over $O$ is available in the environment.

Generally speaking, a tissue P system includes the rules of two types: evolution rules and communication rules. Each elementary membrane usually contains one or more evolution rules, while a communication rule is built between two different cells. In the above definition, $R_{i}(1 \leq i \leq d)$ is finite set of evolution rules in cell $i$, whose rule is of the form $u \rightarrow v, u, v \in O^{*}$. The application of the rule means that $u$ will be evolved to $v$. In most of the existing tissue-like $P$ systems and variants, evolution rule of the form is based on string of objects. However, when we apply it to solve real-world problem, we should design the corresponding evolution rules according to domain knowledge of the real-world problem. The communication rule of the form $(i, u / v, j)$ is called as antiport rule. The communication rule $(i, u / v, j)$ can be applied over two cells labeled by $i$ and $j$ when $u$ is contained in cell $i$ and $v$ is contained in cell $j$. The application of this rule means that the objects of the multisets represented by $u$ and $v$ are interchanged between the two cells. Note that if either $i=0$ or $j=0$ then the objects are interchanged between a cell and the environment. If one of $u$ or $v$ in the above rule is empty, the rule is called as symport rule, for example, $(i, u / \lambda, j)$. The application of the rule means that $u$ will be communicated from cell $i$ to cell $j$.

In tissue $\mathrm{P}$ systems, as usual in the framework of membrane computing, every cell as a computing unit works in a maximally parallel way (a universal clock is 
considered here). In a computing step, each object in a membrane can only be used for one rule (non-deterministically chosen when there are several possibilities), but any object which can participate in a rule of any form must do it, i.e., in each step we can apply a maximal set of rules.

A computation in a tissue-like P system of degree $d$ is a sequence of steps which start with the cells $1, \ldots, d$ containing the multisets $w_{1}, \ldots, w_{d}$ and where, in each step, one or more rules are applied to the current multisets of symbol objects. A computation is successful if and only if it halts. When it halts, it produces a final result in output cell.

\section{An image watermarking algorithm in ridgelet domain}

The image watermarking algorithm presented in this paper is a ridgelet-based watermarking algorithm. The main reason of using ridgelet transform is based on the following fact: compared with wavelet transform, ridgelet transform has better direction property and can effectively extract image features. Especially, its sparse representation ability can concentrate more on the energy of an image after transforming, which is advantageous to better hide the watermark information.

The idea of the ridgelet transform is to map singular lines in an image into singular points by Radon transform and detect the singular points by using wavelet transform, and then use inverse Radon transform to map the singular points into singular lines. More detailed descriptions of ridgelet transform can be found in [30-32]. The watermarking algorithm presented here will employ the blockwise strategy: we divide an image into a series of image blocks, and then achieve ridgelet transform on each image block and embed the watermark into their ridgelet coefficients. For an image block with size $n \times n$, based on the knowledge about ridgelet transform, we can obtain its ridgelet coefficients on $n$ directions after finite ridgelet transform, and the ridgelet coefficients on each direction will exhibit a pyramid structure, whose different layers reflect the different frequency bands of ridgelet coefficients on this direction, such as low-frequency, middlefrequency and high-frequency. Based on the features of ridgelet transform described above, we will consider the following embedding strategy to improve imperceptibility and robustness of the watermarking algorithm designed: (i) for every image block, ridgelet coefficients on the strongest energy direction are selected as the candidates of embedding positions (since the direction contains the strongest energy edges of the image block, its coefficients can tolerate more distortions); (ii) according to human visual system, their low- and middle-frequency coefficients are further selected as final embedding positions. In this paper, watermark information $W$ to be embedded is a randomly generated binary sequence, whose length is equal to the number of image blocks.

\subsection{Watermark embedding}

Fig. 1 shows the procedure of watermark embedding, which uses ridgelet coefficients on the strongest energy direction as embedding positions and an energy modulation method presented in this paper. They are the key points different from the existing watermarking methods. In the following, we will describe in detail the energy modulation method in the ridgelet-based watermarking technique.

Suppose $I$ is a gray image with size $M \times M$. In accordance with block-wise strategy, the image is divided into

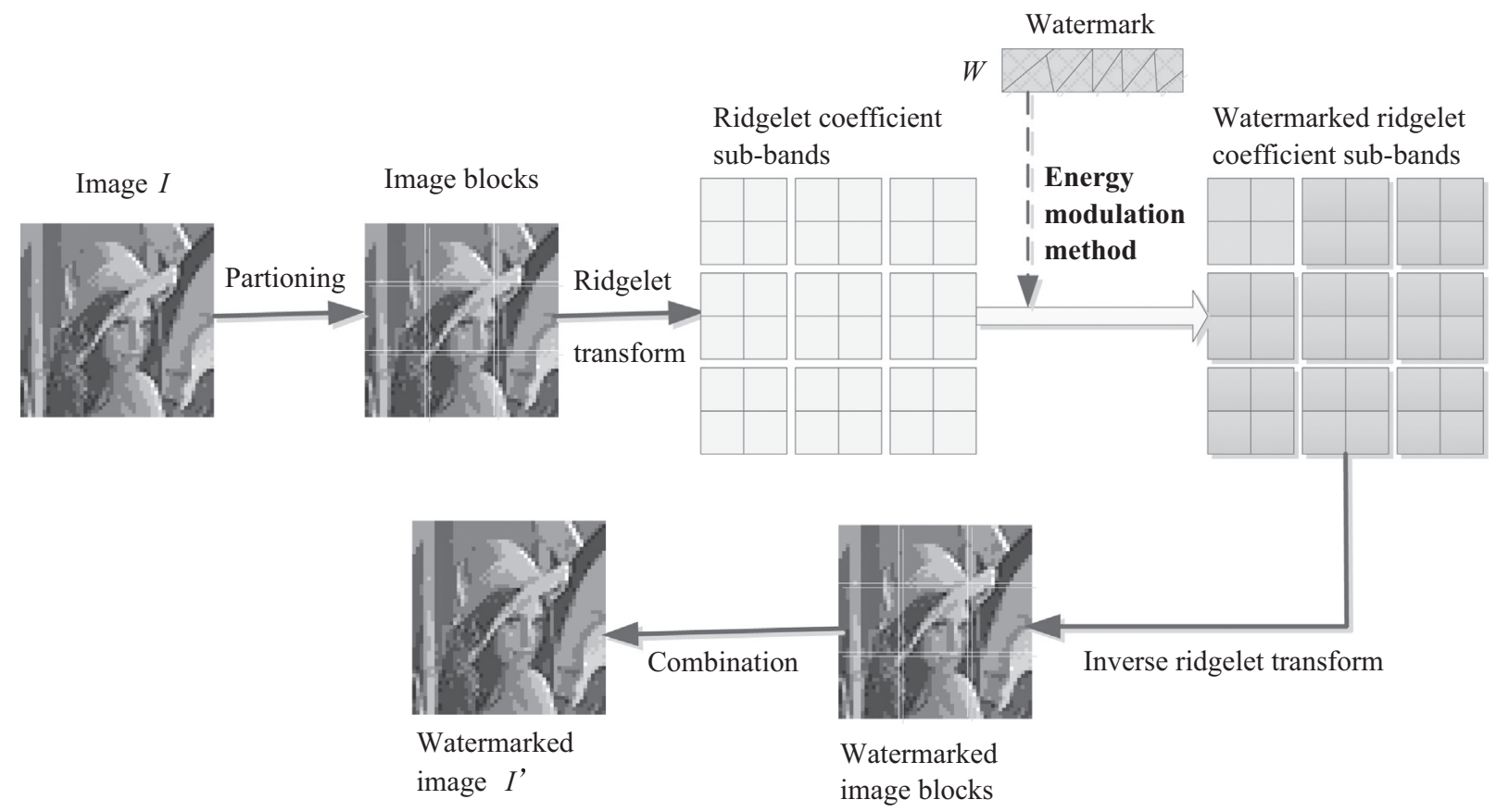

Fig. 1. The embedding procedure of the watermark. 
a series of non-overlapping image blocks with size $n \times n$. Denote $I=\left\{I_{h}, h=1,2, \ldots, m\right\}$, where $I_{h}$ is its $h$ th image block and $m=[(M \times M) /(n \times n)]$. For every image block $I_{h}$, watermark embedding is achieved as follows. First of all, we accomplish ridgelet transform on the image block (wavelet decomposition of $L$ levels is assumed here) to obtain its ridgelet coefficients on $n$ directions, where each direction has $L$ ridgelet coefficient sub-bands. Formally denote $C_{h}=C_{h 1} \cup C_{h 2} \cup \ldots \cup C_{h L}$, where $C_{h l}$ is coefficient set of $l$ th coefficient sub-band. Then, we compute energy of each direction and then find out the strongest energy direction from the $n$ directions, $k_{h}$, formally,

$\left.k_{h}=\max _{1 \leq k \leq n} \sum_{l=1}^{L} \sum_{i=1}^{n} c_{h}^{2}(k, l, i)\right)$,

where $c_{h}^{2}(k, l, i)$ is $i$ th ridgelet coefficient of $k$ th direction in $C_{h l}$. In the following, we select low- and middle-frequency coefficients from the sub-bands of the strongest energy direction $k_{h}$, and the selected coefficients are denoted by $C_{h}=\left\{c_{h 1}, c_{h 2}, \ldots, c_{h J}\right\}$, where $J$ is the number of the selected coefficients. For the strongest energy direction $k_{h}$, we define the following energy index:

$e_{h}=\left(c_{h 1}^{2}+c_{h 2}^{2}+\ldots+c_{h J}^{2}\right)^{1 / 2}$.

In this paper, the energy index of the strongest energy direction $k_{h}$ will be regarded as the object of quantization modulation to embed watermark bit. The modulation rule of the energy index is as follows:

$e_{h}^{\prime}= \begin{cases}\left\lfloor e_{h} / \delta\right\rfloor \cdot \delta+3 \delta / 4 & \text { if } w_{h}=1 \\ \left\lfloor e_{h} / \delta\right\rfloor \cdot \delta+\delta / 4 & \text { if } w_{h}=0\end{cases}$

where $1 \leq h \leq m, \delta$ is a quantization step, $e_{h}^{\prime}$ is the energy index after modulation and $w_{h}$ is watermark bit to be embedded. From the above modulation rule we can see that only one watermark bit is embedded into each image block. In the following, we need to modify every coefficient in low- and middle-frequency sub-bands of the strongest energy direction $k_{h}$ to achieve the above energy modulation. In order to ensure good imperceptibility, we employ a coefficient modification strategy that proportions the modification amount according to absolute value of every ridgelet coefficient, i.e., the coefficients that have larger absolute values will bear larger modification amount, while the coefficients that have smaller absolute values will bear smaller modification amount. For this purpose, the following coefficient modulation formula can be applied:

$c_{h j}^{\prime}=\operatorname{sgn}\left(c_{h j}\right) \sqrt{\frac{c_{h j}^{2}}{\left(c_{h 1}^{2}+c_{h 2}^{2}+\cdots+c_{h j}^{2}\right)}} \cdot \delta$,

where $c_{h j}$ is the original ridgelet coefficient, $c_{h j}^{\prime}$ is the ridgelet coefficient after modulation and $\delta$ is the desired energy index after modulation.

As usual in the existing watermarking methods, we can obtain the watermarked image blocks from the watermarked ridgelet coefficient sub-bands by using inverse ridgelet transform, and then combine the watermarked image blocks to generate the watermarked image $I^{\prime}$.

\subsection{Watermark extraction}

Fig. 2 gives the procedure of watermark extraction. The principle of watermark extraction is illustrated as follows. Suppose $I^{\prime}$ is a gray image with size $M \times M$ to be tested. The image is divided into a series of non-overlapping image blocks with size $n \times n$ in the same way as in embedding algorithm, $I^{\prime}=\left\{I_{h}^{\prime}, h=1,2, \ldots, m\right\}$. We accomplish ridgelet transform on each image block $I_{h}^{\prime}$ to obtain its ridgelet coefficients on $n$ directions, and low- and middlefrequency sub-bands of the strongest energy direction $k_{h}$ will be regarded as extraction positions of the watermark. The selected ridgelet coefficients are formally denoted by $\hat{C}_{h}=\left\{\hat{c}_{h 1}, \hat{c}_{h 2}, \ldots, \hat{c}_{h J}\right\}$, where $J$ is the number of the selected coefficients. In the following, we compute energy index of the coefficient set as follows:

$\hat{e}_{h}=\left(\hat{c}_{h 1}^{2}+\hat{c}_{h 2}^{2}+\ldots+\hat{c}_{h J}^{2}\right)^{1 / 2}$.

According to the energy index, we use the following watermark extraction rule to achieve watermark extraction:

$w_{h}^{\prime}= \begin{cases}1 & \text { if } \hat{e}_{h}-\left\lfloor\hat{e}_{h} / \delta\right\rfloor \cdot \delta \geq \delta / 2 \\ 0 & \text { if } \hat{e}_{h}-\left\lfloor\hat{e}_{h} / \delta\right\rfloor \cdot \delta<\delta / 2\end{cases}$

where $w_{h}^{\prime}$ is the extracted watermark bit, $1 \leq h \leq m$.

From the image watermarking algorithm described above we easily deduce that when $n$ and $L$ are determined as two constants, its computational complexity is $O(m)$ or $\mathrm{O}\left(\mathrm{M}^{2}\right)$.

In the ridgelet-based watermarking algorithm described above, $\delta$ is a watermarking threshold, which plays the role of the embedding strength of the watermark. It is well-known that image blocks may have

Ridgelet coefficient

sub-bands

Test image $I^{\prime} \quad$ Image blocks

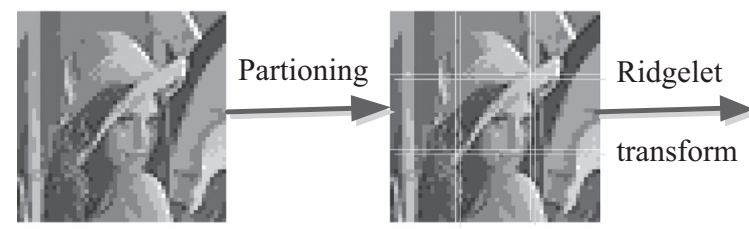

\section{sub-bands}

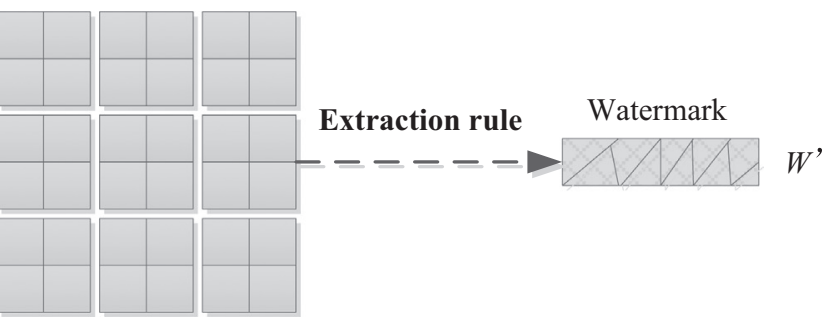

Fig. 2. The extraction procedure of the watermark. 
different local features, so the image blocks should use different thresholds, which imply the idea of adaptive watermarking. In this work, a tissue P system will used to determine the optimal thresholds for the image blocks.

\section{The framework of tissue P systems}

In this section, we will describe a framework of $P$ systems for optimal watermarking, which is based on a tissue P system with symport/antiport rules. The framework includes two kinds of elementary membranes: first kind will use the position-velocity model to evolve the watermarking thresholds of image blocks, while another kind will compute the corresponding performance index. Assume that the number of membranes of first kind is equal to the number of image blocks, whereas second kind contains only one membrane. In addition, the framework also uses communication rules to exchange and share objects among the membranes. Fig. 3 gives the framework of $P$ systems, where elementary membranes (in the dashed box) are $m+1$ parallel computing units. In the following, we will describe in detail each component of the framework.

\subsection{Membrane structure}

Fig. 4 shows the membrane structure of the framework, which consists of $(q+1)$ elementary membranes labeled by $1,2, \ldots, q, q+1$. As usual, the environment is labeled by 0 and is also output membrane of the system. Therefore, when the system halts, objects in the environment are regarded as the final results, i.e., optimal thresholds of image blocks. Based on the used different rules, the $(q+1)$ membranes are classified into two classes: the membrane labeled by $q+1$ is called evaluation membrane, which deals with watermark embedding, watermark extraction and fitness evaluation; other $q$ membranes are called threshold membranes, which evolve the thresholds of image blocks.

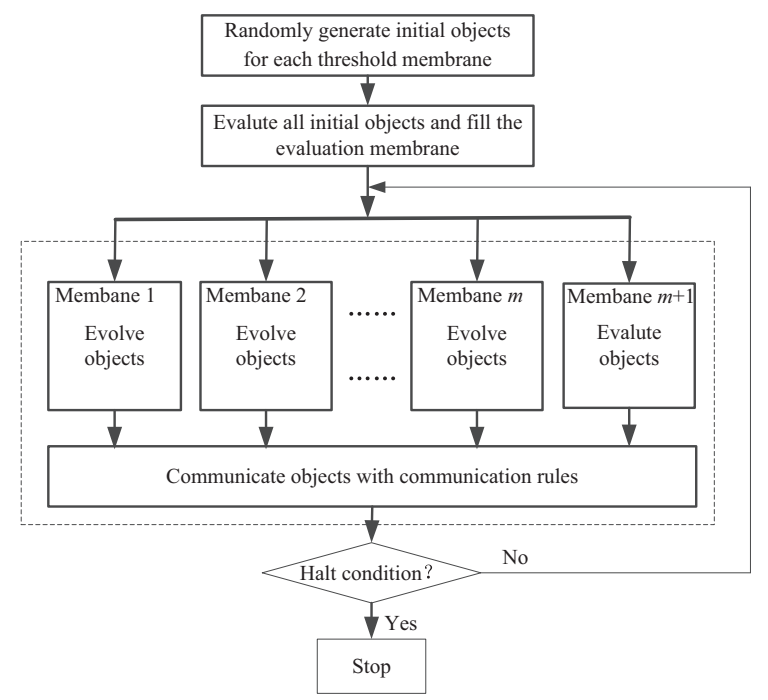

Fig. 3. The framework of $P$ systems applied to solve optimal watermarking problem.

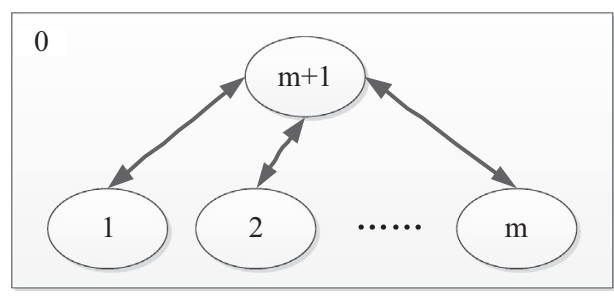

Fig. 4. Membrane structure.

In the framework, the $(q+1)$ membranes as parallel computing units work in a maximally parallel way.

\subsection{Objects}

As usual, each membrane contains an object or several objects. Assume that each threshold membrane in the framework contains $(s+1)$ objects, which are described as follows:

(1) $s$ objects, $X_{i}^{1}, X_{i}^{2}, \ldots, X_{i}^{s}$ : The objects are called threshold objects, where subscript $i$ indicates that the objects are in ith threshold membrane. The purpose is to search for optimal thresholds of image blocks by object's evolution. Here, each object is a triple $X_{i}^{j}=\left(x_{i}^{j}, p_{i}^{j}, f_{i}^{j}\right)$, where $x_{i}^{j}$ is the current position of the object in the positionvelocity model and also expresses the (candidate) threshold of $j$ th object in $i$ th threshold membrane, $p_{i}^{j}$ is the best position of the object so far, and $f_{i}^{j}$ is the corresponding fitness value, $j=1,2, \ldots, s, i=1,2, \ldots, q$.

(2) An object $G_{j}$ : The object is called best position object. Each threshold membrane has only one best position object. The best position object $G_{j}$ is a tuple $G_{i}=\left(g_{i}, f_{i}^{g}\right)$, where $g_{i}$ is best position of all objects in $i$ th threshold membrane so far and $f_{i}^{g}$ is the corresponding fitness value, $i=1,2, \ldots, q$.

In each execution step the evaluation membrane receives, deals with all the objects described above, and also communicates current best object of each threshold membrane into the environment.

Initially, the framework generates $s$ objects for each threshold membrane randomly. The component $x_{i}^{j}$ of each object is a random number generated according to domain knowledge, and set its $p_{i}^{j}$ to be equal to the random number. Then, its fitness value $f_{i}^{j}$ is computed according to the evaluation formula (11) given below. Finally, each threshold membrane creates its object $G_{j}$ according to the object with best fitness.

\subsection{Rules}

The framework uses two different evolution rules in threshold membranes and evaluation membrane, and applies the communication rules to exchange and share objects among them.

\subsubsection{Evolution rules of threshold membranes}

The role of evolution rules in each threshold membrane is to evolve its objects and update its best position object. 
In this paper, the position-velocity model of particle swarm optimization (PSO) [33,34] is used as evolution rule of each threshold membrane which is given by

$v_{i}^{j}=\omega \cdot x_{i}^{j}+c_{1} r_{1}\left(p_{i}^{j}-x_{i}^{j}\right)+c_{2} r_{2}\left(g_{i}-x_{i}^{j}\right)$

$x_{i}^{j}=x_{i}^{j}+v_{i}^{j}$,

where $\omega$ is the inertia weight, $c_{1}$ and $c_{2}$ are the learning factors, and $r_{1}, r_{2} \in(0,1)$ are the two random numbers, $j=1,2, \ldots, s, i=1,2, \ldots, q$.

\subsubsection{Evolution rule of evaluation membrane}

The role of evolution rule in evaluation membrane is to evaluate the objects in threshold membranes. The evolution rule is achieved as follows:

(1) Execute embedding algorithm to generate the watermarked image according to the thresholds of image blocks.

(2) Compute the following peak signal to noise ratio (PSNR) of the watermarked image:

$$
P S N R=10 \cdot \log _{10}\left[\frac{255^{2} \times M^{2}}{\sum_{i=1}^{M} \sum_{j=1}^{M}\left(I(i, j)-I^{\prime}(i, j)\right)^{2}}\right] .
$$

where $I(i, j)$ and $I^{\prime}(i, j)$ are the pixels of the original image and the watermarked image respectively, $i, j=1,2, \ldots, M$. In the existing watermarking techniques, PSNR is commonly used to measure the imperceptibility of a watermarking algorithm.

(3) Obtain $R$ attacked images by using $R$ attack methods to attack the watermarked image.

(4) Execute extraction algorithm to extract the corresponding watermark sequence $W_{r}^{\prime}$ for each attacked image, $r=1,2, \ldots, R$.

(5) Compute the following bit error rate (BER) of the watermarked image:

$B E R_{r}=\frac{\sum_{i=1}^{m} w_{i} \oplus w_{r i}^{\prime}}{m}$,

where $w_{i}$ and $w_{r i}^{\prime}$ are the original watermark bit and the extracted watermark bit respectively, $r=1,2, \ldots, R$.

(6) Compute the fitness value $f_{i}^{j}$ of object $X_{i}^{j}$ :

$f_{i}^{j}=P S N R+\lambda \sum_{r=1}^{R}\left(1-B E R_{r}\right)$

where $\lambda$ is a weight constant.

(7) Update objects $X_{i}^{j}$. If the new value is higher than the original value, then update the value and its position; otherwise it will not be updated.

(8) Update best position object of each threshold membrane, $G_{i}$. If the new value is higher than the original value in $G_{i}$, then update the value and its position; otherwise it will not be updated.

\subsubsection{Communication rules}

The framework has the communication rules of two types:

(1) Antiport rules: (i, $\left.X_{i}^{1} X_{i}^{2} \cdots X_{i}^{s} G_{i} / X_{i}^{\prime 1} X_{i}^{\prime 2} \cdots X_{i}^{\prime s} G_{i}^{\prime}, q+1\right), \quad i=$ $1,2, \ldots, q$. The rule indicates that the objects are communicated in $i$ th threshold membrane and evaluation membrane.

(2) Symport rules: $\left(m+1, X_{1} X_{2} \cdots X_{q} / \lambda, 0\right)$. The rule indicates that the objects are communicated in $i$ th threshold membrane and the environment, where $X_{i}$ is current best object of $i$ th threshold membrane.

\subsection{Halt condition}

In this paper, we employ maximum execution step number as halt condition of the framework, that is, the framework will continue to run until the maximum execution step number is reached. When the system halts, $m$ objects in the environment are the system output, where first component of each output object is the optimal threshold of the corresponding image block.

By analyzing the proposed framework, we easily deduce its computational complexity to be $O(m s R T)$ or $O\left(M^{2} s R T\right)$, where $s$ is the number of objects in each threshold membrane, $R$ is the number of attack methods used and $T$ is the maximum execution step number. Compared with the existing GA-based watermarking methods, the proposed framework has approximately equal computational complexity when they use same parameters (such as same population size, attack methods and maximum generation number).

In the presented watermarking scheme, the optimal problem is viewed as an optimization problem, thus the watermarking framework based on tissue $\mathrm{P}$ systems is used to search for the optimal thresholds for image blocks. Based on the framework, we can describe the optimization procedure of tissue $\mathrm{P}$ systems for the proposed ridgeletbased watermarking algorithm as follows. The framework uses Eq. (11) as the objective function of the optimal watermarking problem, and the thresholds of image blocks are regarded as the parameters to be optimized. The framework first generates initial objects for each threshold membrane, and then successively runs until the halt condition is reached. Finally, we execute the embedding algorithm again to generate the watermarked image according to the obtained optimal thresholds.

\section{Experimental results and analysis}

\subsection{Setup}

In experiments, two image sets are used to evaluate the performance of the proposed watermarking framework. First image set consists of ten standard gray images of size $512 \times 512$, which are commonly used in watermarking literatures, shown in Fig. 5. Second image set contains 146 images, which are from the USC-SIPI image database [35].

The size of image block is set to be $512 \times 512$ due to consideration of the balance of the transform accuracy and visual quality. In the implementation of ridgelet transform, we use the Haar wavelet and achieve wavelet decomposition of four levels on every direction $(L=4)$. Therefore, the number of ridgelet coefficients on every direction is as follows: low-frequency sub-band of 4th layer has 2 ridgelet coefficients, while high-frequency sub-bands of 4 th 

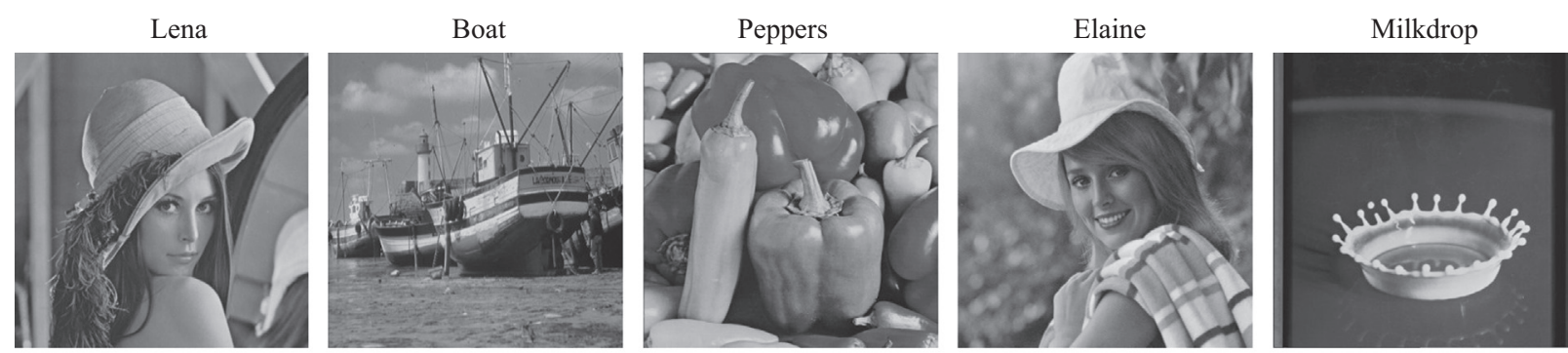

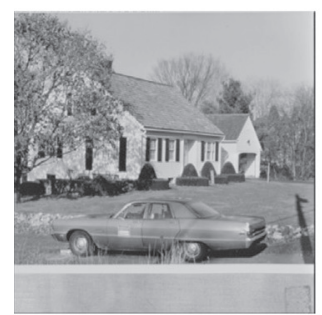

House

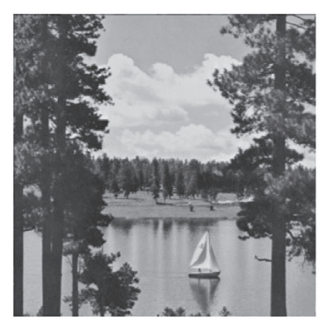

Lake

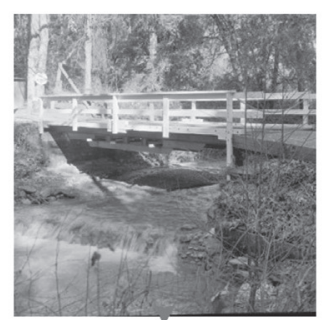

Bridge

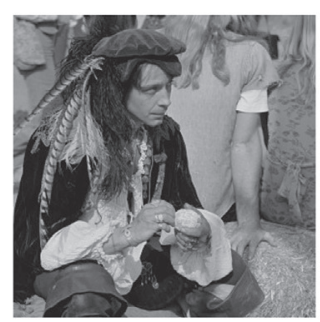

Hunter

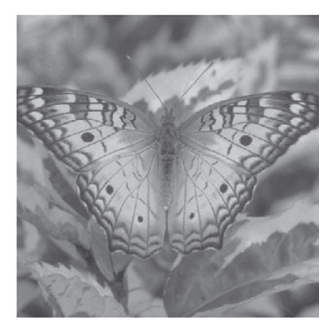

Butterfly

Fig. 5. A small image data set that consists of ten standard gray images of size $512 \times 512$.

layer, 3rd layer, 2nd layer and 1st layer have 2, 3, 5 and 9 ridgelet coefficients respectively.

The parameters of the used tissue P system are set as follows: $s=10$ and $T=200$. In the position-velocity model, $c_{1}=c_{2}=1.0$, and $\omega$ linearly varies from 0.9 to 0.4 . The weight factor in Eq. (11) is set to be $\lambda=5$. In experiments, we employ 6 attack methods $(R=6)$, which are JPEG compression with quality factor (QF) 60 , median filtering $(3 \times 3)$, Gaussian noise with signal to noise ratio (SNR) $20 \mathrm{~dB}$, image scaling (50\%), image cropping (25\%), rotation $\left(75^{\circ}\right)$. Finally, watermark sequence $W$ is a randomly generated binary sequence with 900 bits.

The performance of the proposed framework is investigated by measuring its imperceptibility and robustness against the 6 attacks. For imperceptibility, peak signal to noise ratio (PSNR) is employed to evaluate difference between original images $I$ and the watermarked image $I^{\prime}$ (see Eq. (9)). For robustness, bit error rate (BER) measures difference between the original watermark $W$ and the extracted watermark $W^{\prime}$ (see Eq. (10)). Note that the larger the PSNR, the better the imperceptibility. If a method has a lower BER, it is more robust.

\subsection{Performance test on standard image set}

\subsubsection{Results}

We execute the proposed framework on the standard image set. When the system halts, optimal thresholds of image blocks are generated in the environment. Considering the fact that the framework includes some stochastic mechanisms, we execute the framework on each image 20 times independently. Table 1 gives the means and standard errors of the means (SEM) of the PSNR and BER values of the framework on the ten standard images. Based on the best knowledge of image watermarking, the results indicate good imperceptibility of the proposed framework as well as high robustness against the 6 attacks.
In addition, we also observe such a phenomenon: although these images have different image features, we get approximately equal performances, however, they correspond to completely different thresholds. This observation indicates that for different images, the proposed framework can automatically determine the corresponding optimal thresholds such that a watermarking algorithm has approximately equal performances on them.

\subsubsection{Compared with the GA-based watermarking methods}

For the optimal watermarking problem, GA-based watermarking methods are a class of representative methods in recent years. The proposed framework is compared with several existing GA-based watermarking methods, which are Meng' method [13], Shieh's method [16], Kumsawat's method [18] and Wang's method [20]. Table 2 gives the comparison results of these watermarking methods on Lena image. It is clear from Table 2 that the proposed framework is superior to other methods because of its highest PSNR value, lowest BER value and smallest standard deviation. Likewise, similar results are also obtained in the other images. The results draw a conclusion: benefited from inherent evolution and communication mechanisms of tissue $\mathrm{P}$ systems, the framework can search for global optimal thresholds for a watermarking algorithm. Therefore, the proposed framework is very suitable to solve the optimal watermarking problem.

\subsubsection{Compared with the machine learning-based watermarking methods}

Recently, some watermarking methods based on machine learning (such as artificial neural networks, support vector machines) have been developed and have exhibited the excellent performance. In the experiments, we will compare the proposed framework with several watermarking methods based on machine learning, including Yu's method [36], Tsai's method [12] and Peng's method [15]. The purpose of the comparison is to observe 
Table 1

The average performance results of the proposed framework for 20 runs, including mean values and SEM of PSNR and BER.

\begin{tabular}{|c|c|c|c|c|c|c|c|}
\hline Images & PSNR (db) & JPEG (60) & Median filtering $(3 \times 3)$ & Gaussian noise (20) & Scaling (50\%) & Image cropping (1/4) & Rotation $\left(75^{\circ}\right)$ \\
\hline \multirow[t]{2}{*}{ Lena } & 41.646 & 0.1043 & 0.3012 & 0.1952 & 0.1974 & 0.2358 & 0.2015 \\
\hline & $1.08 \times 10^{-2}$ & $2.36 \times 10^{-4}$ & $2.52 \times 10^{-4}$ & $2.65 \times 10^{-4}$ & $2.21 \times 10^{-4}$ & $3.31 \times 10^{-4}$ & $2.63 \times 10^{-4}$ \\
\hline \multirow[t]{2}{*}{ Boat } & 41.518 & 0.1044 & 0.3014 & 0.1953 & 0.1975 & 0.2359 & 0.2018 \\
\hline & $1.11 \times 10^{-2}$ & $2.49 \times 10^{-4}$ & $2.53 \times 10^{-4}$ & $2.52 \times 10^{-4}$ & $2.33 \times 10^{-4}$ & $3.28 \times 10^{-4}$ & $2.56 \times 10^{-4}$ \\
\hline \multirow[t]{2}{*}{ Peppers } & 41.715 & 0.1042 & 0.3010 & 0.1951 & 0.1971 & 0.2356 & 0.2013 \\
\hline & $1.15 \times 10^{-2}$ & $2.69 \times 10^{-4}$ & $2.74 \times 10^{-4}$ & $2.53 \times 10^{-4}$ & $2.52 \times 10^{-4}$ & $3.35 \times 10^{-4}$ & $2.61 \times 10^{-4}$ \\
\hline \multirow[t]{2}{*}{ Elaine } & 41.653 & 0.1046 & 0.3015 & 0.1955 & 0.1976 & 0.2360 & 0.2017 \\
\hline & $1.09 \times 10^{-2}$ & $2.53 \times 10^{-4}$ & $2.51 \times 10^{-4}$ & $2.46 \times 10^{-4}$ & $2.41 \times 10^{-4}$ & $3.27 \times 10^{-4}$ & $2.53 \times 10^{-4}$ \\
\hline \multirow[t]{2}{*}{ Milkdrop } & 41.526 & 0.1048 & 0.3015 & 0.1957 & 0.1978 & 0.2359 & 0.2019 \\
\hline & $1.13 \times 10^{-2}$ & $2.54 \times 10^{-4}$ & $2.52 \times 10^{-4}$ & $2.61 \times 10^{-4}$ & $2.63 \times 10^{-4}$ & $3.31 \times 10^{-4}$ & $2.64 \times 10^{-4}$ \\
\hline \multirow[t]{2}{*}{ House } & 41.625 & 0.1039 & 0.3011 & 0.1949 & 0.1972 & 0.2356 & 0.2014 \\
\hline & $1.15 \times 10^{-2}$ & $2.58 \times 10^{-4}$ & $2.73 \times 10^{-4}$ & $2.59 \times 10^{-4}$ & $2.65 \times 10^{-4}$ & $3.26 \times 10^{-4}$ & $2.61 \times 10^{-4}$ \\
\hline \multirow[t]{2}{*}{ Lake } & 41.623 & 0.1040 & 0.3012 & 0.1949 & 0.1973 & 0.2356 & 0.2015 \\
\hline & $1.08 \times 10^{-2}$ & $2.49 \times 10^{-4}$ & $2.53 \times 10^{-4}$ & $2.66 \times 10^{-4}$ & $2.55 \times 10^{-4}$ & $3.24 \times 10^{-4}$ & $2.56 \times 10^{-4}$ \\
\hline \multirow[t]{2}{*}{ Bridge } & 41.744 & 0.1042 & 0.3011 & 0.1951 & 0.1972 & 0.2356 & 0.2013 \\
\hline & $1.10 \times 10^{-2}$ & $2.51 \times 10^{-4}$ & $2.53 \times 10^{-4}$ & $2.46 \times 10^{-4}$ & $2.52 \times 10^{-4}$ & $3.30 \times 10^{-4}$ & $2.55 \times 10^{-4}$ \\
\hline \multirow[t]{2}{*}{ Hunter } & 41.593 & 0.1041 & 0.3011 & 0.1950 & 0.1972 & 0.2355 & 0.2012 \\
\hline & $1.14 \times 10^{-2}$ & $2.41 \times 10^{-4}$ & $2.39 \times 10^{-4}$ & $2.42 \times 10^{-4}$ & $2.49 \times 10^{-4}$ & $3.27 \times 10^{-4}$ & $2.56 \times 10^{-4}$ \\
\hline \multirow[t]{2}{*}{ Butterfly } & 41.628 & 0.1042 & 0.3013 & 0.1953 & 0.1974 & 0.2358 & 0.2013 \\
\hline & $1.08 \times 10^{-2}$ & $2.37 \times 10^{-4}$ & $2.38 \times 10^{-4}$ & $2.39 \times 10^{-4}$ & $2.56 \times 10^{-4}$ & $3.29 \times 10^{-4}$ & $2.51 \times 10^{-4}$ \\
\hline
\end{tabular}

Table 2

Comparison results of the proposed method and several GA-based methods on Lena image.

\begin{tabular}{lcccc}
\hline Attack methods & P systems & Shieh [16] & Kumsawat [18] & Wang [20] \\
\hline PSNR (dB) & 41.646 & 40.592 & 41.815 & 41.162 \\
JPEG (QF =60) & 0.1043 & 0.1971 & 0.1086 & 0.1296 \\
Median filtering $(3 \times 3)$ & 0.3012 & 0.4551 & 0.3283 & 0.56 \\
Gaussian noise $(\mathrm{SNR}=20)$ & 0.1952 & 0.3062 & 0.2157 & 0.3598 \\
Scaling (50\%) & 0.1974 & 0.3161 & 0.2035 & 0.2296 \\
Image cropping $(1 / 4)$ & 0.2358 & 0.4147 & 0.3361 & 0.3075 \\
Rotation $\left(75^{\circ}\right)$ & 0.2015 & 0.3359 & 0.2895 & 0.1976 \\
\hline
\end{tabular}

Table 3

Comparison results of the proposed method and several machine learning-based watermarking methods on Lena image.

\begin{tabular}{|c|c|c|c|c|c|}
\hline Attack methods & Proposed methods & Original methods & Yu's method [36] & Tsai's method [12] & Peng's method [15] \\
\hline PSNR (dB) & 41.646 & 41.716 & 41.720 & 41.715 & 41.673 \\
\hline $\mathrm{JPEG}(\mathrm{QF}=60)$ & 0.1043 & 0.3428 & 0.2981 & 0.2866 & 0.1652 \\
\hline Low-pass filtering & 0.2147 & 0.4813 & 0.3852 & 0.3814 & 0.2871 \\
\hline Median filtering $(3 \times 3)$ & 0.3012 & 0.5625 & 0.4189 & 0.4023 & 0.3038 \\
\hline Salt \& peppers noise $(2 \%)$ & 0.1027 & 0.4981 & 0.3526 & 0.2236 & 0.1039 \\
\hline Gaussian noise $(\mathrm{SNR}=20)$ & 0.1952 & 0.4487 & 0.2081 & 0.1968 & 0.1895 \\
\hline Scaling (50\%) & 0.1974 & 0.4462 & 0.7489 & 0.1892 & 0.1962 \\
\hline Image cropping (1/4) & 0.2358 & 0.4923 & 0.7925 & 0.2578 & 0.2325 \\
\hline Rotation $\left(75^{\circ}\right)$ & 0.2015 & 0.3356 & 0.2635 & 0.2215 & 0.2035 \\
\hline
\end{tabular}

whether a conventional watermarking algorithm optimized by the proposed framework can attain or approach the performances of the machine learning-based watermarking methods. Here, the image watermarking algorithm described in Section 3 is regarded as the conventional watermarking algorithm but uses same watermarking parameters for all image blocks, and it is called as original method here. For fair comparison, we determine the watermarking parameters of these watermarking methods to obtain approximate PSNR values.
Table 3 provides the comparison results of these watermarking methods. It can be observed that the performance of the proposed framework significantly exceeds that of the original method. It should be noted that the original method uses the same watermarking algorithm as the proposed framework, but its threshold is determined manually, whereas the proposed framework automatically determines its optimal thresholds. From the comparison results, two conclusions can be drawn: (i) the proposed framework can considerably improve the performance of 
conventional watermarking algorithm; (ii) because the proposed framework can automatically determine optimal thresholds, it can avoid the difficulty of determining optimal thresholds of conventional watermarking algorithm in a manual way.

It is well-known that the existing machine learningbased watermarking methods have high computational complexity since their watermark detectors include a sophisticated training/learning procedure and need long training time (such as artificial networks, support vector machines). In the proposed framework, however, the optimization of watermarking parameters is completed before watermark embedding. Moreover, watermark extraction is achieved by original watermark extraction algorithm. Therefore, compared with the existing machine learning-based watermarking methods, the proposed framework has relatively low computational complexity.

\subsection{Performance test on large image set}

In order to further illustrate the effectiveness of the proposed framework, a large image set (including 146 images) is used in the experiments. The image set is collected from the USC-SIPI image database [35] and is divided into three groups: texture (brodatz textures, texture mosaic, etc.). aerials (high altitude aerial images) and miscellaneous (baboon, the mandril, and other favorites). Note that the image set contains a part of color images, which are converted to gray images in the experiments. The proposed framework is evaluated on the large image set and is compared with four GA-based watermarking algorithms. Table 4 reports the average performances of the watermarking algorithms in 20 consecutive runs. It is evident from this table that the proposed framework is better than other watermarking algorithms in terms of imperceptibility and robustness.
Wilcoxon's rank sum test is a nonparametric statistical significance test for independent samples. The statistical significance test has been conducted at the $5 \%$ significance level in the experiments. We create five groups for the large image set, which are corresponding to the five methods (proposed method, Shieh's method, Kumsawat's method, Meng's method and Wang's method). Each group consists of the PSNR and BER values produced by 20 consecutive runs of the corresponding methods. In order to illustrate that the goodness is statistically significant, we have completed a statistical significance test for the methods. Table 5 gives the $p$-values provided by Wilcoxon's rank sum test for comparison of two groups (one group corresponding to the proposed framework and another group corresponding to some other method) at a time. The null hypothesis assumes that there is no significant difference between the mean values of two groups, whereas there is significant difference in the mean values of two groups for the alternative hypothesis. It is evident from Table 5 that all $p$-values are less than 0.005 ( $5 \%$ significance level). This is a strong evidence against the null hypothesis, establishing significant superiority of the proposed framework.

\section{Conclusions}

The optimal watermarking problem is an interesting open problem in digital watermarking domain so far. A number of attempts to deal with the interesting problem have been made in recent years, such as adaptive watermarking methods, GA-based watermarking methods, machine learning-based watermarking methods. In this paper, we employed a tissue $P$ system with symport/ antiport rules to deal with the problem and developed a novel framework of $P$ systems. A special membrane structure, including several threshold membranes and an evaluation membrane, was designed. The threshold

Table 4

The average performances of the watermarking algorithms on the large image set in 20 consecutive runs.

\begin{tabular}{|c|c|c|c|c|c|}
\hline Attack methods & P systems & Shieh [16] & Kumsawat [18] & Meng [13] & Wang [20] \\
\hline PSNR (dB) & 41.385 & 40.538 & 41.782 & 41.095 & 39.51 \\
\hline $\mathrm{JPEG}(\mathrm{QF}=60)$ & 0.1061 & 0.1992 & 0.1113 & 0.1308 & 0.1079 \\
\hline Median filtering $(3 \times 3)$ & 0.3035 & 0.4585 & 0.3299 & 0.3621 & 0.3088 \\
\hline Gaussian noise $(\mathrm{SNR}=20)$ & 0.1979 & 0.3105 & 0.2192 & 0.2313 & 0.2032 \\
\hline Scaling (50\%) & 0.1996 & 0.3182 & 0.2042 & 0.2082 & 0.1991 \\
\hline Image cropping (1/4) & 0.2372 & 0.4169 & 0.3388 & 0.3747 & 0.2386 \\
\hline Rotation $\left(75^{\circ}\right)$ & 0.2033 & 0.3381 & 0.2913 & 0.2862 & 0.2027 \\
\hline
\end{tabular}

Table 5

The results of $p$-values of Wilcoxon's rank sum test for the proposed framework with other GA-based watermarking methods on the large image set.

\begin{tabular}{|c|c|c|c|c|}
\hline Attack methods & Shieh [16] & Kumsawat [18] & Meng [13] & Wang [20] \\
\hline PSNR (dB) & 0.0041 & 0.0045 & 0.0043 & 0.0036 \\
\hline $\mathrm{JPEG}(\mathrm{QF}=60)$ & $2.4318 \times 10^{-3}$ & $2.3513 \times 10^{-3}$ & $2.4525 \times 10^{-3}$ & $2.3961 \times 10^{-3}$ \\
\hline Median filtering $(3 \times 3)$ & $3.1235 \times 10^{-3}$ & $3.0968 \times 10^{-3}$ & $3.1523 \times 10^{-3}$ & $3.1382 \times 10^{-3}$ \\
\hline Gaussian noise $(\mathrm{SNR}=20)$ & $2.5263 \times 10^{-3}$ & $2.5284 \times 10^{-3}$ & $2.5192 \times 10^{-3}$ & $2.5306 \times 10^{-3}$ \\
\hline Scaling $(50 \%)$ & $2.5291 \times 10^{-3}$ & $2.5276 \times 10^{-3}$ & $2.5235 \times 10^{-3}$ & $2.5268 \times 10^{-3}$ \\
\hline Image cropping (1/4) & $2.4792 \times 10^{-3}$ & $2.4823 \times 10^{-3}$ & $2.4683 \times 10^{-3}$ & $2.4766 \times 10^{-3}$ \\
\hline Rotation $\left(75^{\circ}\right)$ & $2.5033 \times 10^{-3}$ & $2.5085 \times 10^{-3}$ & $2.5118 \times 10^{-3}$ & $2.5047 \times 10^{-3}$ \\
\hline
\end{tabular}


membranes co-evolved watermarking thresholds for image blocks, while the evaluation membrane dealt with the tasks that were associated with watermark embedding, watermark extraction and evaluation of objects in the system. Meanwhile, communication rules between threshold membranes and evaluation membrane were used to exchange and share information. In summary, the proposed framework can effectively deal with the optimal watermarking problem and achieve the excellent performances. This is mainly benefited from the following two mechanisms of tissue P systems: (i) evolution and communication mechanisms (that allow multiple elementary membranes to co-evolve the objects in the system); (ii) parallel computing mechanism (the framework can quickly search for the optimal watermarking thresholds). As we know, some mechanisms that were derived from cell biology have been introduced into P systems and their variants, such as the creation, division and dissolution of the cell. The mechanisms provide new ideas and ways for enhancing the framework or developing new watermarking schemes. Our further work will focus on application of the mechanisms to the framework.

\section{Acknowledgments}

The authors would like to thank the helpful comments and suggestions from the anonymous reviewers.

This work was partially supported by the National Natural Science Foundation of China (Grant no. 61170030), the Chunhui Project Foundation of the Education Department of China (Nos. Z2012025 and Z2012031), and the Sichuan Key Technology Research and Development Program (No. 2013GZX0155), China.

\section{References}

[1] I.J. Cox, M.L. Miller, The first 50 year of electronic watermarking, J. Appl. Signal Process. 2 (2002) 126-132.

[2] L. An, X. Gao, X. Li, D. Tao, C. Deng, J. Li, Robust reversible watermarking via clustering and enhanced pixel-wise masking, IEEE Trans. Image Process. 21 (8) (2012) 3598-3611.

[3] C. Deng, X. Gao, X. Li, D. Tao, Local histogram based geometric invariant image watermarking, Signal Process. 90 (2010) 3256-3264.

[4] H. Peng, J. Wang, Z. Zhang, Audio watermarking scheme robust against desynchronization attacks based on kernel clustering, Multimedia Tools Appl. 62 (3) (2013) 681-699.

[5] L. An, X. Gao, Y. Yuan, D. Tao, C. Deng, F. Ji, Content-adaptive reliable robust lossless data embedding, Neurocomputing 79 (1) (2012) 1-11.

[6] H. Qi, D. Zheng, J. Zhao, Human visual system based adaptive digital image watermarking, Signal Process. 88 (1) (2008) 174-188.

[7] L.H. Chen, J.J. Lin, Mean quantization based image watermarking, Image Vis. Comput. 21 (8) (2003) 717-727.

[8] H. Peng, J. Wang, Z. Zhang, H. Chen, X. Zhang, Energy quantization modulation approach for image watermarking, J. Comput. Inf. Syst. 6 (8) (2010) 2675-2682.

[9] C. Deng, X. Gao, X. Li, D. Tao, A local Tchebichef moments based robust image watermarking, Signal Process. 89 (2009) 1531-1539.
[10] L. An, X. Gao, Y. Yuan, D. Tao, Robust lossless data hiding using clustering and statistical quantity histogram, Neurocomputing 77 (1) (2012) 1-11.

[11] H. Peng, B. Li, X. Luo, J. Wang, Z. Zhang, A learning-based audio watermarking scheme using kernel Fisher discriminant analysis, Digital Signal Process. 23 (1) (2013) 382-389.

[12] H.-H. Tsai, D.-W. Sun, Color image watermark extraction based on support vector machines, Inf. Sci. 177 (2) (2007) 550-569.

[13] F.M. Meng, H. Peng, Z. Pei, J. Wang, An adaptive image watermarking scheme based on support vector machine and genetic algorithm, Pattern Recognit. Artif. Intell. 22 (2) (2009) 312-317.

[14] H. Peng, X. Wang, W. Wang, J. Wang, D.Y. Hu, Audio watermarking approach based on audio features in multiwavelet domain, J. Comput. Res. Dev. 47 (2) (2010) 216-222.

[15] H. Peng, J. Wang, W. Wang, Image watermarking method in multiwavelet domain based on support vector machines, J. Syst. Softw. 83 (8) (2010) 1470-1477.

[16] C.S. Shieh, H.C. Huang, F.H. Wang, J.S. Pan, Genetic watermarking based on transform domain techniques, Pattern Recognit. 37 (3) (2004) 555-565.

[17] H. Peng, J. Wang, Optimal audio watermarking scheme using genetic optimization, Ann. Telecommun. 66 (5-6) (2011) 307-318.

[18] P. Kumsawat, K. Attakitmongcol, A. Srikaew, A new approach for optimization in image watermarking by using genetic algorithm, IEEE Trans. Signal Process. 53 (12) (2005) 4707-4719.

[19] C.H. Huang, J.L. Wu, Fidelity-guaranteed robustness enhancement of blind-detection watermarking schemes, Inf. Sci. 179 (6) (2009) 791-808.

[20] J. Wang, H. Peng, P. Shi, An optimal image watermarking approach based on a multi-objective genetic algorithm, Inf. Sci. 181 (24) (2011) 5501-5514.

[21] Gh. Păun, Computing with membranes, J. Comput. Syst. Sci. 61 (1) (2000) 108-143.

[22] Gh. Păun, G. Rozenberg, A. Salomaa, The Oxford Handbook of Membrane Computing, Oxford University Press, New York, 2010.

[23] H. Peng, J. Wang, M.J. Pérez-Jiménez, H. Wang, J. Shao, T. Wang, Fuzzy reasoning spiking neural P system for fault diagnosis, Inf. Sci. 235 (2013) 106-116.

[24] J. Wang, P. Shi, H. Peng, M.J. Pérez-Jiménez, T. Wang, Weighted fuzzy spiking neural P systems, IEEE Trans. Fuzzy Syst. 21 (2) (2013) 209-220.

[25] J. Wang, H. Peng, Adaptive fuzzy spiking neural P systems for fuzzy inference and learning. Int. J. Comput. Math. 90 (4) (2013) 857-868.

[26] H. Peng, J. Wang, M.J. Pérez-Jiménez, P. Shi, A novel image thresholding method based on membrane computing and fuzzy entropy, J. Intell. Fuzzy Syst. 24 (2) (2013) 229-237.

[27] R. Freund, Gh. Păun, M.J. Pérez-Jiménez, Tissue-like P systems with channel-states, Theor. Comput. Sci. 330 (2005) 101-116.

[28] Gh. Pǎun, M.J. Pérez-Jiménez, A. Riscos-Núňez, Tissue P system with cell division, In: Second Brainstorming Week on Membrane Computing, Sevilla, 2004, Report RGNC 01/2004, pp. 380-386.

[29] D. Díaz-Pernil, M.A. Gutiérrez, M.J. Pérez-Jiménez, A. Riscos-Núňez, A uniform family of tissue P systems with cell division solving 3-COL in a linear time, Theor. Comput. Sci. 404 (2008) 76-87.

[30] E.J. Candes, D.L. Donoho, Ridgelets: a key to higher-dimensional intermittency, Philos. Trans. R. Soc. Lond. A (1999) 2495-2509.

[31] D.L. Donoho, Fast Ridgelet Transforms in Dimension 2, Stanford University, Department of Statistics, Stanford, CA, USA, 1997.

[32] M.N. Do, M. Vetterli, The finite ridgelet transform for image representation, IEEE Trans. Image Process. 12 (1) (2003) 16-28.

[33] R.C. Eberhart, J. Kennedy, Y.H. Shi, Swarm Intelligence, Morgan Kaufmann, San Mateo, CA, 2001.

[34] J. Kennedy, R.C. Eberhart, Particle swarm optimization, in: Proceedings of IEEE International Conference on Neural Networks, vol. 4, Perth, Australia, 1995, pp. 1942-1948.

[35] The USC-SIPI Image Database. 〈http://sipi.usc.edu/database/〉.

[36] P.-T. Yu, H.-H. Tsai, J.-S. Lin, Digital watermarking based on neural networks for color images, Signal Process 81 (3) (2011) 663-671. 